\title{
On the concordance among empirical confusion matrices for visual and tactual letter recognition
}

\author{
MICHAEL J. BRUSCO \\ Florida State University, Tallahassee, Florida
}

\begin{abstract}
In this article, we examine the concordance among 19 empirical confusion matrices for visual and tactual recognition of capital letters of the alphabet. As a measure of concordance, we employed an index based on within-stimulus triads of letters. Unlike correlation measures of agreement that are based on a one-to-one matching of matrix elements, the selected index directly captures the internal structures of the confusion matrices prior to the comparison. Permutation tests revealed statistically significant concordance among 166 of 171 pairs of matrices in the study. Concordance of confusion structure among tactual matrices tended to be somewhat stronger than concordance among the visual matrices.
\end{abstract}

The capital letters of the alphabet have represented the stimulus set for numerous studies of visual and tactual recognition. A common representation of the data from these studies is an $n \times n$ confusion matrix, $\mathbf{A}_{q}$, with elements $a_{i j q}$ representing the proportion of times that letter $j$ was a response to letter stimulus $i$ for matrix $q$ (all letters are considered in most studies, yielding $n=26)$. Not surprisingly, a number of researchers have investigated the concordance (agreement) of confusion matrices obtained from different sources and/or under different experimental conditions. Perhaps the most common measurement for the concordance between two alphabetic confusion matrices is the correlation of the trace elements (correct responses). For example, Mewhort and Dow (1979) demonstrated that there is a rather low correlation between the trace elements of visual confusion matrices obtained by Townsend (1971b) and Gilmore, Hersh, Caramazza, and Griffin (1979). Van der Heijden, Malhas, and van den Roovaart (1984) provided similar correlation comparisons between their visual confusion matrix and those of Townsend (1971b) and Gilmore et al. (1979). Trace element correlation has also been used to compare the agreement between visual and tactual confusion matrices (Craig, 1979; Loomis, 1982; Phillips, Johnson, \& Browne, 1983). There have also been some efforts to compare visual and tactual confusion matrices with respect to the correlation of off-diagonal elements. For example, Loomis (1982, p. 46, Table 5) reported correlation coefficients for symmetrized confusion errors for three visual and five tactual confusion matrices. Pairwise correlation coefficients among these matrices

I thank Jim Townsend, Larry Hubert, and two anonymous reviewers for helpful comments on earlier versions of this article. These comments led to significant improvements in both content and exposition. Correspondence should be addressed to M. J. Brusco, Department of Marketing, College of Business, Florida State University, Tallahassee, FL 323061110 (e-mail: mbrusco@cob.fsu.edu). ranged from .38 to .83 . Phillips et al. (1983, p. 247, Table 2) obtained similar findings using the asymmetric confusion frequencies.

Previously reported correlation comparisons of visual and tactual confusion matrices have certainly provided some insight with respect to the concordance of such matrices. In this article, our goal was to expand the knowledge base by providing additional data regarding the concordance of confusion structures among a broad set of empirical matrices. Specifically, this study is important in two aspects: (1) the large number of empirical matrices included in the analysis, and (2) the employment of a measure of concordance that captures the internal confusion structure of each matrix.

\section{Limitations of Correlation Analysis for Confusion Matrices}

Hubert (1978) observed that a potential limitation of correlation-type indices for comparing two proximity matrices is that they are computed on the basis of one-to-one products of corresponding elements of matrices. Although correlation analysis does have a role in the comparison of confusion matrices, it should be recognized that this method is designed to test only for the presence of linear association in such matrices, and internal structural similarities between matrices might be overlooked. We propose that comparisons based on the within-row (i.e., within-stimulus) patterns of confusion elements are especially important and provide a viable alternative (or supplement) to correlation analyses. For example, when considering the presentation of the letter $\mathrm{L}$ in visual and tactual recognition, it might be interesting to determine whether or not the pattern of incorrect responses to that stimulus is similar across the empirical studies in the literature. Although a method has been developed for facilitating comparisons on the basis of the within-stimulus structures of confusion matrices (Hubert, 1987), its exiguous deployment in the literature necessitates a detailed presentation of the procedure. 


\section{A Measure of Within-Stimulus Concordance Between Pairs of Confusion Matrices}

Hubert $(1978,1987)$ has presented a number of concordance indices that capture the internal structural characteristics of proximity matrices. One such index is based on within-stimulus triads of objects. To present this index, we define $S=\left\{\mathbf{A}_{1}, \mathbf{A}_{2}, \ldots, \mathbf{A}_{Q}\right\}$ as a set of $Q$ confusion matrices. For each two-way array in $S$, a three-way array is established on the basis of the following relationships:

$$
\begin{aligned}
b_{h i j q}= \begin{cases}\operatorname{sign}\left(a_{h j q}-a_{h i q}\right) & \text { for distinct } h, i, j \\
0 & \text { otherwise, }\end{cases} \\
\text { where } \operatorname{sign}(x)=\left\{\begin{aligned}
+1 & \text { if } x>0 \\
0 & \text { if } x=0 . \\
-1 & \text { if } x<0
\end{aligned}\right.
\end{aligned}
$$

On the basis of this definition, the value of $b_{h i j q}$ is 1 $(-1)$ if the element in row $h$, column $j$ of matrix $q$ is larger (smaller) than the element in row $h$, column $i$ of matrix $q$. If the elements in columns $i$ and $j$ of row $h$ are equal, then $b_{h i j q}=0$. For each pair of matrices - say, $\mathbf{A}_{q}$ and $\mathbf{A}_{r}$ - an index based on the within-stimulus order relationships of confusions is then computed as follows:

$$
\begin{aligned}
\Omega_{q r}= & \frac{\sum_{h, i, j} b_{h i j q} b_{h i j r}}{\sum_{h, i, j}\left|b_{h i j q} b_{h i j r}\right|} \\
& \text { for } q=1, \ldots, Q-1, r=q+1, \ldots, Q .
\end{aligned}
$$

The numerator of Equation 2 represents the number of within-stimulus triads that are consistent for matrices $\mathbf{A}_{q}$ and $\mathbf{A}_{r}$ minus the number of within-stimulus triads that are inconsistent for the pair of matrices. The denominator of Equation 2 is the total number of within-stimulus consistencies plus the total number of within-stimulus inconsistencies. This denominator is one of five possible alternatives offered by Hubert (1987, pp. 274-276) and is the one he selected for his analysis of auditory confusion matrices published by Miller and Nicely (1955). The $\Omega_{q r}$ indices can also be described within the context of the ratio of the consistencies to inconsistencies. For example, $\Omega_{q r}$ values of $0, .333, .5, .6$, and .666 correspond to consistency-inconsistency ratios of 1:1, 2:1, 3:1, 4:1, and 5:1, respectively.

Significance tests for the within-stimulus concordance index can be obtained by using permutation methods. The implementation of permutation-based methods for significance testing dates back to the pioneering work of R. A. Fisher (1935) and Pittman (1937), and such methods were introduced to the field of psychology by Edgington $(1964,1966,1969)$. A nice review pertaining to the use of permutation tests as an alternative to $t$ tests of correlation coefficients is provided by Hayes (1996). Hubert (1987) has developed a permutation test for the within-stimulus triad concordance of confusion matrices $\mathbf{A}_{q}$ and $\mathbf{A}_{r}$. To describe this test, we define $\Psi$ as the set of all $n$ ! permutations, and $\psi \in \Psi$ as a permuta- tion selected from that set. Furthermore, we define $\psi(k)$ as the object in position $k$ of the permutation $\psi$. A distribution for the test statistic, $\Omega_{q r}$, is obtained by selecting a large number of permutations from $\Psi$. Each selected permutation is used to provide a relabeling of the rows and columns of one of the matrices (herein, we relabel $\mathbf{A}_{r}$ ), and the within-stimulus index is computed for that relabeling as follows:

$$
\Omega_{q r}(\psi)=\frac{\sum_{h, i, j} b_{h i j q} b_{\psi(h) \psi(i) \psi(j) r}}{\sum_{h, i, j}\left|b_{h i j q} b_{\psi(h) \psi(i) \psi(j) r}\right|} .
$$

For small confusion matrices $(n \leq 10)$, it is possible to compute Equation 3 for all $n$ ! permutations, thus generating the entire distribution for the concordance statistic (this is sometimes referred to as an exact permutation test). For larger values of $n$, such as the case of $n=26$ for alphabetic confusion matrices, complete enumeration of the distribution is computationally infeasible. Therefore, we resort to random sampling of permutations from the set of all permutations, $\Psi$ (this is sometimes referred to as an approximate permutation test). In this article, we will compute Equation 3 for 99,999 randomly selected permutations to generate a reference distribution for the concordance statistic. If $\Omega_{q r}$ is sufficiently extreme in this distribution, then the null hypothesis of no withinstimulus structural concordance between the two matrices is rejected.

\section{Numerical Demonstrations of the Within- Stimulus Concordance Index}

Our first demonstration of the concordance index uses the synthetic $3 \times 3$ confusion matrices displayed in the first panel of Table 1. A computation of the Pearson correlation coefficient for the off-diagonal elements of matrices $\mathbf{A}_{t}$ and $\mathbf{A}_{u}$ in Table 1 yields $r=.98$. The second panel of Table 1 illustrates the computation of the within-stimulus concordance index for matrices $\mathbf{A}_{t}$ and $\mathbf{A}_{u}$. We will consider the triad of $h=1, i=2$, and $j=3$ (i.e., we will examine row 1 and columns 2 and 3 of matrices $\mathbf{A}_{t}$ and $\mathbf{A}_{u}$ ). For matrix $\mathbf{A}_{t}$, we have $b_{123 t}=$ $\operatorname{sign}\left(a_{13 t}-a_{12 t}\right)=\operatorname{sign}(20-2)=+1$, whereas for ma$\operatorname{trix} \mathbf{A}_{u}$ we have $b_{123 u}=\operatorname{sign}\left(a_{13 u}-a_{12 u}\right)=\operatorname{sign}(20-$ $3)=+1$. Because the $b_{h i j q}$ values for this triad are both +1 , we have a consistency between the two matrices with respect to this triad. In other words, when Stimulus 1 was presented, three was given more frequently as a response than was two for each of the two matrices. Now consider the triad corresponding to $h=2, i=1$, and $j=3$. For matrix $\mathbf{A}_{t}$, we have $b_{213 t}=\operatorname{sign}(3-1)=$ +1 , whereas for matrix $\mathbf{A}_{u}$ we have $b_{213 u}=\operatorname{sign}(1-$ $2)=-1$. This is an example of an inconsistency between the two matrices. When Stimulus 2 was presented, three was a more frequent response than was one in matrix $\mathbf{A}_{t}$, but three was a less frequent response than was one in matrix $\mathbf{A}_{u}$. In the computation of the index, $\Omega_{t u}$, $b_{213 t} b_{213 u}=+1(-1)=-1$ reduces the index value because of this inconsistency. 
Table 1

Synthetic $3 \times 3$ Confusion Matrices, $A_{t}$ and $A_{u}$, Along With Within-Stimulus Concordance Computations and Permutation Test Summary Statistics

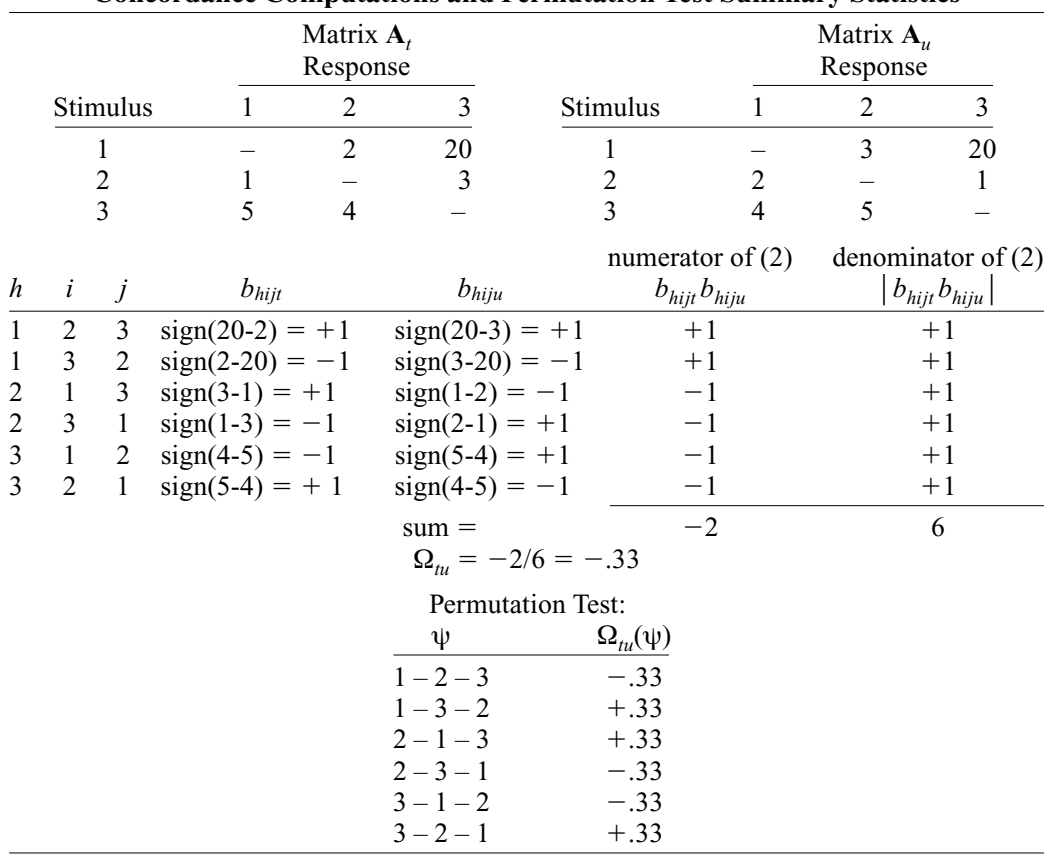

For matrices $\mathbf{A}_{t}$ and $\mathbf{A}_{u}$, the within-stimulus concordance index is computed as $\Omega_{t u}=-.33$. An exact permutation test can easily be conducted for this pair of matrices by computing Equation 3 for all 3 ! $=3 \times 2 \times 1=$ 6 permutations of the rows and columns of matrix $\mathbf{A}_{u}$. The resulting concordance index for each permutation is provided in the third panel of Table 1 . The index value of $\Omega_{t u}=-.33$, which corresponds to the identity permutation (1-2-3) of the rows and columns of matrix $\mathbf{A}_{u}$, falls in the lower end of the distribution. This finding suggests that matrices $\mathbf{A}_{t}$ and $\mathbf{A}_{u}$ do not share similar withinstimulus patterns of confusion. Moreover, the fact that $\Omega_{t u}$ is negative suggests that there is a tendency for the two matrices to exhibit dissimilar patterns of withinstimulus confusion. Thus, despite the large positive correlation between matrices $\mathbf{A}_{t}$ and $\mathbf{A}_{u}$, we clearly do not observe a high degree of within-stimulus concordance.

It is also possible to observe weak correlation between two matrices, despite a strong within-stimulus patterning of the confusion elements. This situation is illustrated using the two matrices (matrices $\mathbf{A}_{x}$ and $\mathbf{A}_{y}$ ) in Table 2. The Pearson correlation coefficient between the offdiagonal elements of the two matrices in Table 2 is $r=$

Table 2

Synthetic $3 \times 3$ Confusion Matrices With Low Correlation but Strong Within-Row Concordance

\begin{tabular}{ccccccccc}
\hline & \multicolumn{3}{c}{ Matrix } & $\mathbf{A}_{x}$ Response & & & \multicolumn{3}{c}{ Matrix $\mathbf{A}_{y}$ Response } \\
\cline { 2 - 4 } \cline { 6 - 8 } Stimulus & 1 & 2 & 3 & & Stimulus & 1 & 2 & 3 \\
\hline 1 & - & 2 & 6 & & 1 & - & 2 & 3 \\
2 & 5 & - & 1 & & 2 & 6 & - & 4 \\
3 & 3 & 4 & - & & 3 & 1 & 5 & - \\
\hline
\end{tabular}

.31. However, a consistent patterning of within-stimulus entries is readily apparent for matrices $\mathbf{A}_{x}$ and $\mathbf{A}_{y}$. In the first row, we observe consistency because the value in column 3 is larger than the value in column 2 for both matrices (i.e., $6>2$ and $3>2$ ). In the second row, the value in column 1 is larger than the value in column 3 for both matrices (i.e., $5>1$ and $6>4$ ). Finally, in the third row, the value in column 2 is larger than the value in column 1 for both matrices (i.e., $4>3$ and $5>1$ ). Because of this perfect consistency in each row, the resulting concordance index is $\Omega_{x y}=1$.

The two examples in this section demonstrate the importance of the concordance index as a supplement to correlation analysis when comparing confusion matrices. In the first example, a large positive correlation between matrices $\mathbf{A}_{t}$ and $\mathbf{A}_{u}$ is observed, but the two matrices do not share similar within-stimulus patterning with respect to responses to stimuli. In the second example, a researcher might conclude no agreement between matrices $\mathbf{A}_{x}$ and $\mathbf{A}_{y}$ because of a weak correlation. However, the within-stimulus concordance analysis of these two matrices reveals perfect agreement.

\section{Analysis of Empirical Matrices}

Table 3 provides the citations for 19 empirical confusion matrices (11 visual and 8 tactual) for uppercase letters of the alphabet, which were collected from the literature. Most of these matrices were originally presented in row-standardized form (i.e., the raw confusion frequencies in each row were divided by the total number of times that the stimulus was presented). All matrices that were not originally presented in this form were row- 
Table 3

Visual and Tactual Confusion Matrices Among Capital Letters of the

Alphabet Included in the Concordance Study

\begin{tabular}{llll}
\hline \multicolumn{1}{c}{ Visual Confusion Matrices } & & \multicolumn{1}{c}{ Tactual Confusion Matrices } \\
\cline { 1 - 1 } 1. Pew and Gardner (1965) & & 12. Loomis (1974), stationary \\
2. D. F. Fisher et al. (1969), $200 \mathrm{msec}$ & & 13. Kikuchi et al. (1979) \\
3. D. F. Fisher et al. (1969), $400 \mathrm{msec}$ & & $\begin{array}{l}\text { 14. Craig (1979) } \\
\text { 4. Townsend (1971b), Condition I }\end{array}$ & 15. Johnson and Phillips (1981) \\
5. Townsend (1971b), Condition II & & 16. Loomis (1982), tactual \\
6. Townsend (1971a), MJ & & 17. Phillips et al. (1983), tactual \\
7. Townsend (1971a), VF & & 18. Vega-Bermudez et al. (1991), active \\
8. Gilmore et al. (1979) & & 19. Vega-Bermudez et al. (1991), passive \\
9. Loomis (1982), visual & & \\
10. Phillips et al. (1983), visual & & \\
11. van der Heijden et al. (1984) &
\end{tabular}

normalized by the author prior to analysis, in order to represent all 19 matrices on a comparable basis. The Pearson correlation coefficients and within-stimulus concordance index (see Equation 2) for each pair of matrices is displayed in Table 4. For convenience, the pairwise indices are partitioned by horizontal and vertical lines that help to distinguish between the visual and tactual matrices.

The Pearson correlation coefficients ranged from .097 to .906 . Tests of significance for the correlation coefficients were performed using matrix permutation methods (Hubert, 1987, Chap. 4). Specifically, for each pair of matrices, 99,999 random relabelings of the rows and columns of one of the matrices in the pair were used to produce a distribution for the correlation coefficient. Only the smallest correlation coefficient, corresponding to matrices $\mathbf{A}_{5}$ and $\mathbf{A}_{12}$, was not statistically significant at $\alpha=.01$. An approximate permutation test, using 99,999 random permutations to generate a sampling dis- tribution for the concordance statistic, was completed for each pair of matrices. These tests resulted in the rejection of the null hypothesis $(\alpha=.01)$ of no agreement for 166 of the 171 matrix pairs. In fact, for $91.8 \%$ of the matrix pairs, the observed statistic was greater than each of the 99,999 random relabelings. Together, the correlation and concordance results strongly support a finding of similar confusion structures among the empirical matrices.

In many cases, the correlation and concordance tests provided similar evidence regarding the agreement of matrix pairs. For example, the largest correlation coefficient (.906) in Table 4 and the largest concordance index (.690) were realized for the same pair of matrices $\left(\mathbf{A}_{18}\right.$ and $\mathbf{A}_{19}$ ). There was also general correspondence between the smaller correlation coefficients and the smaller concordance indices, which can be observed by studying the relationship of Townsend's (1971b) Condition II matrix (matrix $\mathbf{A}_{5}$ ) with the tactual matrices $\left(\mathbf{A}_{12}-\mathbf{A}_{19}\right)$.

Table 4

Within-Stimulus Triad Indices and Correlations Among Pairs of Confusion Matrices

\begin{tabular}{|c|c|c|c|c|c|c|c|c|c|c|c|c|c|c|c|c|c|c|c|}
\hline & 1 & 2 & 3 & 4 & 5 & 6 & 7 & 8 & 9 & 10 & 11 & 12 & 13 & 14 & 15 & 16 & 17 & 18 & 19 \\
\hline 1 & - & .468. & .351 & .462 & .483 & .406 & .417 & .331 & .448 & .241 & .432 & 165 & . 160 & .434 & 336 & .417 & .262 & .421 & .483 \\
\hline 2 & .292 & - & .827 & .633 & .405 & .715 & .531 & .353 & .445 & .340 & .495 & 248 & .205 & 440 & .313 & 348 & 279 & 81 & .393 \\
\hline 3 & .382 & .599 & - & .516 & .346 & .728 & .425 & .281 & .354 & .294 & .472 & 204 & .163 & .364 & .251 & .268 & 192 & 290 & 309 \\
\hline 4 & .301 & .428 & .379 & - & .533 & .681 & .642 & .485 & .531 & .449 & .585 & 292 & .315 & .509 & .358 & .446 & .348 & 399 & .448 \\
\hline 5 & .227 & .277 & .298 & .399 & - & .437 & .337 & .256 & .339 & .223 & .335 & .097 & .158 & .337 & .199 & .213 & .197 & .211 & .289 \\
\hline 6 & .373 & .469 & .457 & .471 & .344 & - & .695 & .395 & .451 & .379 & .603 & .267 & .289 & .483 & .309 & .365 & .256 & .354 & .370 \\
\hline 7 & .378 & .439. & .416. & .524 & .247 & .641 & - & .422 & .485 & .444 & .622 & .249 & .376 & .482 & .309 & .438 & .345 & . 407. & 390 \\
\hline 8 & .392 & . 411. & .452. & .416 & .197 & .444. & .496 & - & .621 & .315 & .580 & .372 & .424 & .564 & .444 & .562 & .365 & .450 & .478 \\
\hline 9 & .270 & 281 & .331 & .378 & .161 & .378 & .496 & .394 & - & .565 & .570 & .450 & .482 & .589 & .603 & .726 & .540 & .648 & .691. \\
\hline 10 & .247 & .298 & .236 & .377 & .269 & .434 & .387 & .287 & .416 & - & .406 & .369 & .378 & .407 & .460 & .532 & .459 & .474 & .444 \\
\hline 11 & .414 & .379 & .424 & .482 & .229 & .497 & .520 & .461 & .383 & .368 & - & .312 & .435 & .550 & .383 & .454 & .295 & .446 & .457 \\
\hline 12 & .272 & .330 & .346 & .334 & .134 & .318 & .407 & .337 & .374 & .325 & .314 & - & .382 & .390 & .432 & .363 & .375 & .411 & .371 \\
\hline 13 & .266 & 267 & .238 & .316 & .087 & .355 & .461 & .373 & .485 & .406 & .329 & .381 & - & .470 & .478 & .462 & .443 & .450 & .394 \\
\hline 14 & .384 & 275 & .368 & .364 & .150 & .460 & .482 & .447 & .395 & .268 & .466 & .362 & .365 & - & .444 & .608 & .405 & .645 & .653 \\
\hline 15 & .356 & .301 & .334 & .346 & .172 & .434 & .487 & .455 & .502 & .327 & .425 & .413 & .458 & .445 & - & .572 & .657 & .556 & .549 \\
\hline 16 & .398 & 288 & .343 & .366 & .081 & .389 & .465 & .427 & .510 & .350 & .438 & 342 & .439. & .478 & .514 & - & .582 & .789 & 806 \\
\hline 17 & .295 & .269 & .228 & .370 & .147 & .404. & .432 & .370 & .426 & .317 & .389 & 328 & .423 & .405 & . 562 & 495 & - & .529 & .534 \\
\hline 18 & .384 & .302 & .340 & .270 & -.008 & .381 & .450 & .375 & .491 & .266 & .406 & 363 & .371 & .488 & .527 & 617 & .512 & - & .906 \\
\hline 19 & .391 & .270 & .316 & .323 & .105 & .372 & .437 & .417 & .518 & .307 & .422 & .396 & .410 & .527 & .551 & .605 & .523 & 690 & - \\
\hline
\end{tabular}

Note-The elements above the main diagonal are the Pearson correlation coefficients, whereas the elements below the main diagonal are concordance indices. The bold figures indicate that the $p$ value for the test statistic is greater than .01 . 
Notice, however, that the concordance index test was less apt to reject the null hypothesis of no agreement for these matrix pairs. All five of the matrix pairs for which the null hypothesis of no within-stimulus concordance could not be rejected involved matrix $\mathbf{A}_{5}$; however, the permutation test of the correlation coefficient failed to reject for only one of these pairs.

There were also a number of instances in which the within-stimulus concordance measure and the correlation coefficient were somewhat contradictory. For example, matrix $\mathbf{A}_{1}$ realizes its strongest correlation with matrices $\mathbf{A}_{5}$ and $\mathbf{A}_{19}(r=.483)$. However, the concordance index for matrices $\mathbf{A}_{1}$ and $\mathbf{A}_{19}$ (.391) is much larger than is the concordance index for matrices $\mathbf{A}_{1}$ and $\mathbf{A}_{5}$ (.227). In fact, this latter index is the smallest concordance index that matrix $\mathbf{A}_{1}$ shares with any other matrix. Thus, examination of the correlation coefficient alone would suggest that matrix $\mathbf{A}_{1}$ 's agreement with matrices $\mathbf{A}_{5}$ and $\mathbf{A}_{19}$ is equally strong. However, the concordance analysis shows that the within-stimulus agreement between matrices $\mathbf{A}_{1}$ and $\mathbf{A}_{19}$ is relatively strong, whereas the within-stimulus agreement between matrices $\mathbf{A}_{1}$ and $\mathbf{A}_{5}$ is relatively weak. There are also some cases in which the correlation between a pair of matrices is relatively weak but the within-stimulus concordance between those matrices is strong. A good example is the pair corresponding to matrices $\mathbf{A}_{7}$ and $\mathbf{A}_{12}$. These two matrices are not highly correlated $(r=.249)$, but their concordance index is a healthy .407.

Perhaps the most salient finding from this investigation is that the empirical tactual matrices exhibited a much greater concordance of within-stimulus confusion structure than did the empirical visual matrices, despite comparable correlation measures for these two categories. The mean within-stimulus concordance index among pairs of tactual matrices was .464 , whereas the corresponding mean for visual matrices was only .381 . This distinction was much less evident in the correlation analyses, in which the averages of the correlation coefficients across the visual matrix pairs and tactual matrix pairs were .471 and .465 , respectively.

Although the disparity between visual and tactual within-stimulus structures is partially attributable to poor concordance between matrix $\mathbf{A}_{5}$ and all other matrices, there is additional evidence of stronger concordance among tactual matrices. One piece of evidence stems from the fact that there were 15 visual matrix pairs with within-stimulus concordance indices less than .3, whereas the smallest index among pairs of tactual matrices was .328. The tactual matrices also had a greater proportion of pairs with concordance indices greater than .5. Only 7\% (4 of 55) of the visual pairs yielded indices greater than .5 , whereas $36 \%$ (10 of 28 ) of the tactual pairs exceeded this level.

For both the visual and tactual matrices, some of the largest within-stimulus concordance indices were observed between pairs of matrices from the same study. For example, the two largest indices among visual ma- trices corresponded to matrices $\mathbf{A}_{2}$ and $\mathbf{A}_{3}$ (.599) from D. F. Fisher, Monty, and Glucksberg's (1969) study and matrices $\mathbf{A}_{6}$ and $\mathbf{A}_{7}$ (.641) from Townsend's (1971a) study. In a similar manner, the largest concordance index among tactual matrices occurred between matrices $\mathbf{A}_{18}$ and $\mathbf{A}_{19}(.690)$ from Vega-Bermudez, Johnson, and Hsiao's (1991) investigation of active and passive touch. Relative to the visual matrices, there was much stronger concordance of confusion structure across tactual matrices from different studies. For example, the index values among all pairs of tactual matrices $\mathbf{A}_{15}-\mathbf{A}_{19}$ exceeded .495 , and the tactual matrix from Loomis's (1982) study had a concordance index above .6 when paired with either of the matrices from the Vega-Bermudez et al. studies.

Our evaluation of concordance of confusion structures also tends to support previous research with respect to the similarity between visual and tactual recognition. Among the pairs of matrices formed by one visual and one tactual matrix, the average concordance index was .347 (despite a number of rather poor index values associated with matrix $\mathbf{A}_{5}$ ). Visual matrices $\mathbf{A}_{7}$ and $\mathbf{A}_{9}$ provided especially strong concordance with the tactual matrices. This finding is particularly noteworthy for matrix $\mathbf{A}_{7}$ (Townsend, 1971a), which was not obtained within the context of comparing visual and tactual recognition.

\section{Conclusions}

There are a number of possible methods for comparing the agreement of confusion matrices. Perhaps the most common method is to apply separate correlation analyses of the main diagonal and off-diagonal elements. In this article, we have implemented an alternative approach for studying the off-diagonal elements. Specifically, we employed a method for measuring concordance among confusion structures that carefully follows the illustration by Hubert (1987, Chap. 5). One of the advantages of this approach is that it captures the internal structure of confusion when obtaining the index. Upon implementation of this procedure, we observed generally strong concordance among the confusion structures of a large number of visual and tactual matrices pertaining to recognition of capital letters of the alphabet. Concordance was especially strong among tactual matrices, even across studies by different authors.

\section{REFERENCES}

Craig, J. C. (1979). A confusion matrix for tactually presented letters. Perception \& Psychophysics, 26, 409-411.

Edgington, E. S. (1964). Randomization tests. Journal of Psychology, 57, 445-449.

EDGINGTON, E. S. (1966). Statistical inference and nonrandom samples. Psychological Bulletin, 66, 486-487.

EDGington, E. S. (1969). Approximate randomization tests. Journal of Psychology, 72, 143-149.

Fisher, D. F., Monty, R. A., \& Glucksberg, S. (1969). Visual confusion matrices: Fact or artifact? Journal of Psychology, 71, 111-125.

Fisher, R. A. (1935). The design of experiments. London: Oliver \& Boyd.

Gilmore, G. C., Hersh, H., Caramazza, A., \& Griffin, J. (1979). 
Multidimensional letter similarity derived from recognition errors. Perception \& Psychophysics, 25, 425-431.

HAYES, A. F. (1996). Permutation test is not distribution-free: Testing $\mathrm{H}_{0}: \rho=0$. Psychological Methods, 1, 184-198.

HuberT, L. J. (1978). Generalized proximity function comparisons. British Journal of Mathematical \& Statistical Psychology, 31, 179192.

HuBERT, L. J. (1987) Assignment methods in combinatorial data analysis. New York: Marcel Dekker.

Johnson, K. O., \& Phillips, J. R. (1981). Tactile spatial resolution: I. Two-point discrimination, gap detection, grating resolution, and letter recognition. Journal of Neurophysiology, 46, 1177-1191.

Kikuchi, T., Yamashita, Y., Sagawa, K., \& WaKe, T. (1979). An analysis of tactile letter confusions. Perception \& Psychophysics, 26, 295-301.

Loomis, J. M. (1974). Tactile letter recognition under different modes of stimulus presentation. Perception \& Psychophysics, 16, 401-408.

Loomis, J. M. (1982). Analysis of tactile and visual confusion matrices. Perception \& Psychophysics, 31, 41-52.

MEWHORT, D. J. K., \& Dow, M. L. (1979). Multidimensional letter similarity: A confound with brightness? Perception \& Psychophysics, 26, 325-326.

Miller, G. A., \& Nicely, P. E. (1955). Analysis of perceptual confusions among some English consonants. Journal of the Acoustical Society of America, 27, 338-352.
Pew, R. W., \& Gardner, G. T. (1965). [Unpublished data]. University of Michigan, Ann Arbor.

Phillips, J. R., Johnson, K. O., \& Browne, H. M. (1983). A comparison of visual and two modes of tactual letter resolution. Perception \& Psychophysics, 34, 243-249.

PitTman, E. J. G. (1 $\overline{93} 7)$. Significance tests which may be applied to samples from any population: II. The correlation coefficient test. Journal of the Royal Statistical Society, 4, 225-232.

Townsend, J. T. (1971a). Alphabetic confusion: A test of models for individuals. Perception \& Psychophysics, 9, 449-454.

TownsEnd, J. T. (1971b). Theoretical analysis of an alphabet confusion matrix. Perception \& Psychophysics, 9, 40-50.

van der Heijden, A. H. C., Malhas, M. S. M., \& van den Roovaart, B. P. (1984). An empirical zinterletter confusion matrix for continuousline capitals. Perception \& Psychophysics, 35, 85-88.

Vega-Bermudez, F., Johnson, K. O., \& HsiaO, S. S. (1991). Human tactile pattern recognition: Active versus passive touch, velocity effects, and patterns of confusion. Journal of Neurophysiology, $\underline{\mathbf{6 5}}_{2}$ $\underline{531-546 .}$

(Manuscript received February 4, 2003; revision accepted for publication May 20, 2003.) 